\title{
Reflets
}

Revue ontaroise d'intervention sociale et communautaire

\section{Modèle d'apport de services en santé communautaires aux francophones du Sud de l'Ontario : Entrevue avec Janet Rajroop}

\section{Lynn Casimiro}

Volume 8, numéro 1, printemps 2002

La réadaptation : son visage français en Ontario

URI : https://id.erudit.org/iderudit/026383ar

DOI : https://doi.org/10.7202/026383ar

Aller au sommaire du numéro

Éditeur(s)

Reflets : Revue ontaroise d'intervention sociale et communautaire

ISSN

1203-4576 (imprimé)

1712-8498 (numérique)

Découvrir la revue

Citer cet article

Casimiro, L. (2002). Modèle d'apport de services en santé communautaires aux francophones du Sud de l'Ontario : Entrevue avec Janet Rajroop. Reflets, 8(1), 173-176. https://doi.org/10.7202/026383ar

Tous droits réservés (C) Reflets : Revue ontaroise d'intervention sociale et communautaire, 2002
Ce document est protégé par la loi sur le droit d'auteur. L'utilisation des services d'Érudit (y compris la reproduction) est assujettie à sa politique d'utilisation que vous pouvez consulter en ligne.

https://apropos.erudit.org/fr/usagers/politique-dutilisation/ 


\section{Modèle d'apport de services en santé communautaires aux francophones du Sud de l'Ontario}

\section{Entrevue avec Janet Rajroop}

Directrice des services aux clients du CASC de Scarborough

\section{Lynn Casimiro}

Conseillère en développement pour le Centre national de formation en santé

Par cette entrevue, nous souhaitions mieux comprendre comment les francophones vivant en situation minoritaire en Ontario, accèdent aux services de santé communautaire dans leur langue maternelle. Nous avons pris connaissance d'un modèle administratif appliqué dans les Centres d'accès aux services communautaires (CASC) de la région de Toronto visant l'accès à des services en français. Janet Rajroop est bien ancrée dans le milieu communautaire puisqu'elle a occupé pendant plusieurs années un poste administratif pour les pourvoyeurs de services désignés. Elle occupe maintenant le poste de directrice des services aux clients du CASC de Scarborough et a participé à l'élaboration de ce nouveau modèle administratif.

Cette courte entrevue qui s'est déroulée en anglais, le 28 février 2002 , se veut une simple description du modèle. Elle ne juge pas l'application du modèle ni son efficacité. Cela n'est pas le but de cet article. Nous espérons que les lecteurs de Reflets trouveront le modèle intéressant et s'en inspireront pour améliorer l'accès en français à des services communautaires dans leur région. 
Reflets: Qu'est qu'un CASC?

J.Rajroop : Le rôle du CASC est de gérer les informations et les réquisitions des clients nécessitant des soins communautaires. Les CASC dépendent des pourvoyeurs de services désignés pour dispenser les traitements ou les soins requis. C'est la responsabilité du pourvoyeur de trouver les ressources nécessaires pour fournir les services demandés dans l'une ou l'autre des deux langues officielles.

Les réquisitions proviennent en majeure partie des milieux hospitaliers. Les CASC s'occupent d'informer les responsables des milieux hospitaliers au sujet des services offerts. Si les réquisitions stipulent le besoin de services en français ou si le client demande des services en français, le coordonnateur s'assurera d'en faire la demande au pourvoyeur de service désigné.

Reflets: Quel est la structure des CASC de Toronto?

J.Rajroop : Au total, il y a 43 CASC en Ontario. Dans la région de Toronto il y en a six, définis par les délimitations de l'ancienne ville de Toronto : Scarborough, East York, North York, Toronto, Ville de York et Étobicoke. Ils sont financés par le ministère de la Santé de l'Ontario. Chacun des CASC reçoit une allocation budgétaire qu'il doit respecter. Il est donc important de dépenser les fonds publics de façon judicieuse. Les CASC sont indépendants les uns des autres mais ils ont intérêt à collaborer lorsqu'il s'agit d'un projet pouvant bénéficier au groupe. Cela permet une utilisation sensée des fonds disponibles.

Reflets : Comment les patients accèdent-ils à des services en langue française?

J.Rajroop: Chacun des CASC doit offrir des services en français tel que mandaté par le ministère de la Santé.Toutefois un modèle spécial a été développé en 2000 entre 4 CASC de la région de Toronto. Les CASC de Scarborough, EstYork, Toronto et la ville deYork se sont entendus pour partager un coordonnateur régional assigné à la gestion des clients de langue française. La personne choisie pour occuper ce poste opère depuis Scarborough et coordonne la livraison des services, dispense les informations requises et établit les plans de traitement et ce, en français. 
Prenons, par exemple, une personne de la région de Toronto qui souhaite obtenir des services en français et qui demande de l'information sur les ressources francophones disponibles. Elle peut appeler directement le coordonnateur et obtenir toutes les précisions requises. Ce dernier devient donc le facilitateur de cet appel pour le CASC de Toronto, même s'il est en poste à Scarborough. Le coordonnateur doit être au courant des principaux programmes de sa région et même de l'ensemble de la province. Il peut également agir en tant que traducteur ou interprète pour le personnel anglophone.

Ce modèle a été créé pour soulager certains problèmes d'accès qui existent pour les francophones. Il y a donc un point de service pour tous les clients francophones de la région de Toronto. Les autres CASC de la région de Toronto ou d'ailleurs en Ontario peuvent accéder à ce service et, selon le besoin, le coordonnateur doit gérer les appels. Ce sont toutefois les 4 CASC mentionnés qui partagent le coût de cette ressource humaine.

Reflets : Avez-vous de la difficulté à trouver du personnel qualifié pouvant desservir la clientèle francophone de Toronto?

J.Rajroop : Le coordonnateur régional désigné pour fournir les informations en français est déjà un atout indispensable. Le premier contact est donc toujours en français. Par la suite les clients francophones sont vus par des coordonnateurs bilingues. Dans la région de Scarborough, les clients francophones sont souvent bilingues et peuvent donc communiquer avec nous en anglais. Ils réussissent à obtenir l'information nécessaire. Toutefois, tous les efforts sont faits pour que le coordinateur soit francophone.

Reflets: Quelles sont les particularités de la population francophone de Toronto?

J.Rajroop: Dans mon CASC de Scarborough nous devons desservir une clientèle multiculturelle affichant au-delà de 40 langues parlées. Nous sommes toujours à la recherche de personnel pour rencontrer les besoins multiculturels de notre clientèle. Si nous n'avons pas la ressource nécessaire nous avons recours à des traducteurs professionnels. 
Nous avons noté que plusieurs de nos francophones de la région sont originaires des pays francophones de l'Afrique et qu'il ne sont pas nécessairement des Franco-Ontariens de souche. De plus, les francophones parlent souvent une troisième langue.

Reflets : Avez-vous quelque chose à ajouter?

J.Rajroop: Le modèle administratif que nous avons créé est novateur et a été bien reçu par les instances gouvernementales concernées.Tout projet qui a pour but d'augmenter les services aux clients des minorités francophones est toujours bienvenu et il nous fait plaisir de l'offrir.

Reflets: Je vous remercie d'avoir pris le temps de nous parler et de nous avoir présenté votre modèle de livraison de services. 